\title{
Transformaciones familiares: desafío para la educación del siglo XXI
}

\author{
Gladys Jadue ${ }^{1}$ \\ Universidad Austral de Chile
}

\begin{abstract}
Este artículo analiza algunos factores sociales que han incrementado las transformaciones en la familia, aumentando el riesgo en los hijos de bajo rendimiento, desadaptación, y problemas emocionales y conductuales, los cuales pueden dar lugar al abandono de las aulas. Entre estos factores se encuentran la mayor supervivencia de los individuos, una mayor movilidad geográfica, la inclusión de la mujer en el campo laboral, así como el incremento de familias uniparentales y los niveles de pobreza. Se propone la implementación en las escuelas de mecanismos protectores para los alumnos provenientes de familias disfuncionales, utilizando para ello talleres de desarrollo personal que propicien el autoconocimiento y el de los demás, la interacción humana y la buena relación con los familiares.
\end{abstract}

Palabras clave: familias transformadas, familias disfuncionales, dificultades escolares.

\section{Family changes: A challenge for education in the XXI century}

The article analyses some social factors that increase the possibility of family changes that enlarge children's low achievement and failure, emotional disturbance and behavioral problems at school. Among these factors are the increased life expectancy and geographical mobility, the increased participation of women in the labor force, as well as uniparental families and poverty. It is argued that through the creation of personal development workshops with children from dysfunctional families it is possible to build supporting networks that allow for a good school performance in these children. These workshops should promote understanding of self and others, human interaction and good relationships with family members.

Key words: dysfunctional families, school underachievement.

1 Doctora en psicología educacional por la Universidad de Kentucky (USA). Magíster en psicología y D.E.S.S. en psicología clínica por la Universidad París V Sorbona. Es profesora titular de la Universidad Austral de Chile. Correo electrónico: gjadue@uach.cl. 

Dentro de un mundo globalizado, en Latinoamérica, al igual que en muchos otros países, se desarrolla el proceso de modernización. Modernización se refiere a los avances tecnológicos de racionalización y a la transformación del trabajo y de la organización, e incluye muchos otros cambios: el cambio de los caracteres sociales y de las biografías, de los estilos de vida, de las formas de amar, de las estructuras de influencia y de poder, de las formas políticas de opresión y de participación, de las concepciones de la realidad y de las normas cognoscitivas. Todos estos cambios transforman las fuentes de la certeza de que se nutre la vida.

En la actualidad, la diversidad de la vida familiar es considerable, hasta el punto que no parece que exista una norma estándar de forma familiar ni de un prototipo de familia contemporánea. Los mayores niveles de educación y la mayor incorporación de la mujer al mundo laboral han cambiado en muchas mujeres su rol en la familia.

Dentro y fuera de la familia el ser humano, el individuo, se convierte en actor de la segurización de su existencia y de la planificación de su vida para acceder al mercado laboral, con lo que da prioridad a sus propias metas y define su identidad propia en términos de atributos personales más que de identificación con su grupo familiar y social.

Si en el proceso de transformación de las sociedades contemporáneas no ha habido una convergencia en un único modelo de familia, tal como las teorías sociológicas de los años sesenta habían postulado, ello indica que la familia está ligada a los procesos de transformación de la cultura contemporánea. Si en el presente podemos hablar al mismo tiempo de una cultura global junto a una gran diversidad de formas culturales, la 
familia participa tanto de esta multiplicidad de sentidos como de la relativa homogeneización de comportamientos.

La familia ha dejado de ser el punto de referencia estable de un mundo definido por la movilidad geográfica y social de los individuos, y participa de la misma fragmentación de fluidez de la sociedad contemporánea, ya que como parte integrante de los diferentes procesos históricos no es ni un receptor pasivo de los cambios sociales ni un elemento inmutable en un mundo en constante transformación.

La familia actual vive definida por la diversidad y también por la cohesión y la solidaridad. El individuo tiene, en mayor medida que en el pasado, capacidad de elección en cuanto a sus formas de vida y de convivencia.También han cambiado las relaciones personales que configuran la familia.

La diversidad familiar es considerable hasta el punto que no parece que exista una norma estándar de familia ni un prototipo de familia contemporánea. El ideal de familia nuclear cerrada se ha desmoronado. Sin embargo, esto no significa necesariamente una pérdida del rol de la familia y del parentesco. Las relaciones de parentesco, lejos de dejar de existir, parece que toman nuevas fuerzas y se convierten en un valor sólido a partir de esta incertidumbre (Iglesias de Ussel, 1998).

Aún cuando la globalización conlleva una serie de ventajas respecto de la integración económica y política, genera temor en relación a la pérdida de los valores culturales y de la identidad, principio fundamental de la lógica tradicional. El cambio, desde una sociedad "segura" a una "plural, llena de incertidumbres" requiere de una constante reflexión y aspectos tan centrales como la familia nuclear y patriarcal están dando paso a "una gran diversidad de formas familiares"(Flecha \& Tortajada, 1999). 
Transformaciones familiares: desafio para la educación del siglo XXI

Entre los factores que hacen más complicada la convivencia familiar en la actualidad es posible distinguir (Florenzano, 1998):

1. La mayor supervivencia de sus miembros individuales, lo que hace que los adultos convivan por períodos más prolongados. Hoy en día, una madre pasa más años interactuando con sus hijas mujeres que niñas, ya que al aumentar la expectativa de vida especialmente en la mujer, una madre anciana interactúa con su hija adulta durante muchos años.

2. La mayor movilidad geográfica, que hace que las familias se separen en unidades nucleares y pierdan la posibilidad de apoyo mutuo que ofrecía la familia extendida tradicional.

3. Las construcciones urbanas pequeñas, que dificultan tener espacio para la familia trigeneracional y hacen que los abuelos tengan que vivir solos o en hogares para la tercera edad.

4. La entrada masiva de la mujer al mundo laboral, que hace que más frecuentemente ambos miembros de la pareja trabajen, lo que hace que muchos roles tradicionalmente femeninos deban ser compartidos por ambos cónyuges.

5. Las mayores distancias intraurbanas, lo que trae consigo que se gaste más tiempo en traslados desde y hacia el hogar, lo que disminuye el tiempo de interacción familiar durante la semana. Cada vez son menos las familias que comparten el almuerzo cotidiano y más aún, la cena familiar no es posible en muchos casos.

El estudio del Programa de las Naciones Unidas para el Desarrollo "Nosotros los chilenos: un desafío cultural" (PNUD, 2000), muestra que el $70 \%$ de las personas entrevistadas otorgan importancia fundamental a la familia, el cónyuge y especialmente los hijos, lo que significa que gran parte de los chilenos valoriza la familia como esencial en sus vidas personales. Sin embargo, esto no significa que la familia tradicional no esté sufriendo un proceso de cambio.

Por otra parte, el XVII Censo Nacional de Chile (2002), muestra un notable cambio en la familia chilena: cada vez son más las mujeres 
que son jefes de hogar; más de un tercio de los hogares chilenos están encabezados por una mujer; aumentaron de un 26,9\% en 1992 a un $33,3 \%$ en el 2002 .

\section{Influencia familiar en el desarrollo emocional y psicosocial de los hijos}

En los últimos quince años, Chile ha desarrollado un acelerado proceso de modernización en lo económico, lo social y lo cultural. Estos cambios, al promover el individualismo, provocan una crisis dentro de la familia. Asimismo, fomentan un tipo de vida más hedonista donde el placer se separa de los vínculos tradicionales y se proyecta en gran medida en la libertad y autonomía individual. Sin embargo, el costo personal es, entre otros, mayor vulnerabilidad ante los síntomas y las enfermedades relacionadas con la tensión emocional (Triandis, Bontempo, Villareal, Asai \& Luca, 1988).

La ansiedad en el niño y el adolescente es uno de los síntomas más comunes de la tensión emocional. Hasta cierto punto es lógico atribuir estos signos, entre otros factores, a la rapidez con que cambia la sociedad, al fomento de la competencia y del individualismo en todos los ámbitos sociales (familia, escuela, comunidad), lo que en muchas personas genera ansiedad, conflictos, frustración y otros problemas emocionales. En general, se produce una sensación incómoda de tensión y aprensión que por su duración hacen que el sujeto se perciba a sí mismo muy intranquilo, lo que puede traducirse en problemas de conducta y/o del rendimiento en la escuela.

Las relaciones restringidas al interior de la familia provocan ansiedad, especialmente en los hijos (Doll \& Lyon, 1998). Un ambiente familiar cargado de conflictos interpersonales provoca déficit en el desarrollo emocional y genera distintos niveles de ansiedad y de desórdenes ansiosos (Hansen, Sanders, Scott \& Last, 
1998; Johnson, 1998), especialmente si los padres sufren de ansiedad y/o depresión, y si se producen conflictos y discordias en forma sostenida.

Las experiencias familiares negativas pueden influir en la autopercepción del niño, en su capacidad de control emocional y conductual, y éstas cogniciones pueden contribuir a que se desarrolle y mantenga la ansiedad (King, Mietz \& Ollendik, 1995).

Niños criados bajo condiciones de abuso físico y emocional tienen más posibilidades de desarrollar trastornos psicológicos y problemas conductuales (Kolvin, Miller, Fleeting \& Kolvin, 1988). Los conflictos maritales y familias disfuncionales son predictores de desajustes emocionales en los hijos (Jadue, 1999). Hijos provenientes de familias con altos niveles de conflicto pueden no aprender las habilidades sociales como la negociación y el compromiso (Doll \& Lyon, 1998).

\section{Familia y educación}

La familia también ejerce una poderosa influencia en la educación de los hijos. Los padres juegan un rol fundamental en el proceso enseñanza-aprendizaje, ya que si los padres se preocupan de la educación de sus hijos y colaboran con los profesores, los niños presentan buen rendimiento y se adaptan fácilmente a la escuela. Por este motivo, numerosos estudios indican la necesidad de incorporar a los padres de familia a la tarea que cumple la escuela (De Castro, 1996; Gligo, 1996; Kotliarenco, Cáceres \& Castro, 1994; Mc Lanahan,1985; Ramey \& Campbell, 1984).

En Chile se han desarrollado programas educativos que reconocen el rol de la familia como fundamental en la educación de sus hijos. El Programa Padres e Hijos desarrollado por el Centro de Investigación y Desarrollo de la Educación (CIDE), estimula especialmente las 
interacciones de los padres con sus hijos preescolares para facilitar su incorporación a la escuela y busca crear las condiciones necesarias para obtener la participación de los padres a través de la confianza en sus propias capacidades educativas, ya que suponen que los padres son capaces de enfrentar la educación de sus hijos (Fuenzalida \& Jiménez, 1994). Asimismo, el Centro de Estudios de Atención al Niño y la Mujer, a través de los Centros Comunitarios de Atención Preescolar, incorpora el desarrollo de habilidades humanas y familiares y la participación activa y directa de las madres que asisten a dichos centros, estimulando el nivel y la calidad de la interacción intrafamiliar de las mujeres, de los niños y de la diada madre-hijo (Kotliarenco, Cáceres \& Cortéz, 1995).

Cuando se incorpora el apoyo familiar a la educación de los niños, los resultados son significativamente más eficaces que cuando se trabaja solamente con alumnos (Jadue, 1996a; Wang, 1995). La implicación de la familia en la tarea educativa comprende no sólo una participación activa de los padres en los proyectos educativos de la escuela, sino además la concepción de los padres como mediadores del aprendizaje (Arancibia, Herrera \& Strasser, 1997; Williams \& Chaukin, 1989). Este compromiso implica compartir la información, asistir como voluntario a la escuela y ayudar a los hijos en la casa. Los padres, como primeros profesores de los niños, juegan un papel muy significativo en el proceso de aprendizaje y de socialización.

Un ambiente familiar cálido y sin discordias, en el que los padres se desempeñen de una manera competente y estimuladora, lleva en sí una orientación ética de los padres, que influye en su percepción y en su autoevaluación, como asimismo en la valoración de sus hijos y de los demás, influyendo en el tipo de interacciones intrafamiliares y su inserción en una red social más amplia (Villalón, De Castro \& Streeter, 1998). El apoyo de los padres a los hijos aparece determinado por una valoración de las propias capacidades para apoyar este proceso, independientemente del nivel socioeconómico y cultural al que pertenecen (Mc Allister, 1990), pero también se asocia a las características de los 
padres y de los hijos, al contexto familiar y a las actitudes de los profesores hacia los padres y hacia los niños (Villalón et al., 1998).

El alumno enfrenta constantemente situaciones académicas que debe comparar con sus propios medios. Cuando su evaluación de las demandas de la escuela lo llevan a concluir que son más de lo que él puede rendir, afronta una situación de peligro, de humillación, a veces suficiente para justificar un rechazo a la escuela. En general, la humillación originada por pequeños fracasos es transitoria. Sin embargo, algunos estudiantes sienten sus fracasos más que otros; llegan a la escuela después de haber sufrido fracasos en su hogar (Jackson \& Frick, 1998).

Resultados de varios estudios muestran que a los niños que tienen una buena relación con sus padres les tiende a ir mejor en el colegio. Las experiencias familiares se asocian con la adaptación a la escuela, incluyendo la relación madre-hijo y las interacciones del niño o adolescente con los miembros de su familia. De tal manera que las dimensiones positivas o negativas de su relación con cada padre son predictores de la adaptación a la escuela, como también lo son las percepciones que los jóvenes tienen del grado en que reciben el apoyo que necesitan de parte de los integrantes de su núcleo familiar (Lau \& Leung, 1992). Los procesos afectivos intrafamiliares, la ayuda otorgada por los padres para un buen desempeño escolar, el refuerzo dado a las notas y las expectativas de un buen rendimiento del hijo, colaboran para que éstos últimos se desempeñen mejor en la escuela (Pitiyanuwat \& Reed, 1994).

Sin embargo, comparando las diferencias entre hijos de familias intactas con aquellos provenientes de familias de padres separados, se observa que los primeros presentan mejores calificaciones escolares y los segundos problemas de conducta y más propensión a fracasar en la escuela (Florenzano, 1998). Además, en un estudio destinado a conocer las percepciones de los profesores respecto de 
sus alumnos de padres separados, el 92,8\% de los maestros entrevistados afirman que existen diferencias en el comportamiento emocional entre los niños de padres separados y aquellos que pertenecen a familias intactas. Aunque, respecto del rendimiento académico, sólo un $7,14 \%$ de los profesores entrevistados indicó que la separación de los padres tiene efectos negativos en el rendimiento escolar (Santelices, Ossandón \& De la Barrera, 2000).

Es evidente que el tipo de familia a la que el estudiante pertenece constituye un elemento clave para surgir tanto en lo psicosocial como en lo material. Una familia constituida por ambos progenitores, con apoyo mutuo, estable y funcional en sus relaciones intrafamiliares, ayuda al progreso económico y psicosocial de las personas que la componen, y favorece el desarrollo emocional.

En general, los niños crecen sin problemas de ajuste cuando tienen una buena relación con un solo padre, a diferencia de lo que ocurre cuando crecen en un hogar con dos padres pero que se caracteriza por la discordia y el descontento (Rutter,1983). Asimismo, un padre inaccesible, hostil y rechazante puede causar más daño que un padre ausente (Hetherington, 1980).

En este contexto, la valoración que el estudiante hace de sí mismo (autoestima) y las cogniciones acerca de sí mismo (autoconcepto) están en la base del desarrollo de la competencia emocional.

La competencia emocional y social es la habilidad para comprender, dirigir y expresar los aspectos emocionales de nuestra propia vida, de tal manera que seamos capaces de manejar las tareas cotidianas como aprender, establecer relaciones interpersonales y sociales, resolver los problemas de cada día y adaptarnos a las complejas demandas del crecimiento y del desarrollo. Esta competencia incluye varios aspectos, tanto de la comprensión y de la expresión emocional como del manejo de la experiencia emocional interna y de la conducta expresiva. La 
competencia en el aprecio emocional incluye el reconocimiento y la comprensión, tanto de la expresión corporal como de la experiencia emocional interna y de los demás, y una correcta apreciación de aspectos emocionales relevantes en el contexto social. Estas habilidades están incluidas en lo que Goleman (1996) y Salovey y Sluyter (1997) denominan inteligencia emocional, y Gardner (1995) las comprende en lo que denomina inteligencia intrapersonal e inteligencia interpersonal.

Las alteraciones en la competencia emocional y social, inseparables del desarrollo emocional, afectan la conducta y el aprendizaje en la escuela, lo que se traduce en bajo rendimiento y/o problemas de conducta y riesgo de fracaso y de deserción (Jadue, 2002).

\section{Familia, educación y pobreza}

Los efectos acumulados de la pobreza influyen directamente en la vulnerabilidad física y psicosocial del niño que crece y se desarrolla en medio de factores ambientales adversos (UNESCO-UNICEF, 1996; UNESCO, 1997). El nivel educativo de la madre, poderoso predictor del rendimiento escolar, es más bajo en las familias pobres (Broman, Bien \& Shaugenessy, 1985).

Los padres de bajo nivel socioeconómico y cultural interactúan escasamente en destrezas relacionadas con el éxito escolar y utilizan estrategias poco efectivas para enseñar a sus hijos, aunque valoren la educación y deseen que ellos tengan un buen rendimiento en la escuela (Jadue, 1996a). Sin embargo, no es sólo la falta de recursos económicos lo que determina la falta de apoyo a los hijos, sino también los recursos personales de los padres para enfrentar esta situación y su interés activo y positivo hacia sus hijos, lo que se produce especialmente si existen buenas relaciones de pareja, apoyo familiar y de la escuela (Osborn, 1990). Es posible distinguir tres 
factores cotidianos de protección: la seguridad, la filiación y la afectividad (Amar \& Abello, 1998), entendidos como todos aquellos elementos y circunstancias que la familia de bajo nivel socioeconómico y cultural utiliza para proteger a los niños de carencias concretas, a través de los cuales se apoyan y defienden mutuamente.

El bajo nivel educativo de los padres, la pobreza y las dificultades escolares de los hijos son factores mutuamente relacionados. Se ha establecido en muchos países que los niños que presentan bajo rendimiento en la escuela provienen desproporcionadamente de familias de bajo nivel socioeconómico y cultural (Alvarez, 1986; Beech, 1985; Halpern, 1986; Hollsteiner \& Tacon, 1983; Montenegro, 1989; UNESCO/UNICEF, 1996).

Asimismo, una de las atribuciones que los profesores de los niños provenientes de familias de bajo nivel socioeconómico y cultural hacen del bajo rendimiento y del fracaso en la escuela, se ubica exclusivamente en el plano familiar y del niño. Estos profesores piensan que el déficit para el aprendizaje y para la adaptación a la escuela se debe a la falta de interés y de apoyo de parte de la familia y al bajo nivel cultural de los padres o a los problemas económicos y sociales de la familia (Filp, 1995).

La familia de bajo nivel socioeconómico y cultural, aunque valore la educación, no tiene capacidad ni interés para favorecer la educación de sus hijos, los apoyan poco en sus afectos y algunas además presentan problemas sociales como alcoholismo, delincuencia y hogares destrozados.

Los estudiantes provenientes de familias uniparentales, con madre sola, tienen rendimiento escolar bajo y alto riesgo de abandono escolar y de experiencias negativas en la escuela (Suet, como se cita en Luisi $\&$ Santelices, 2000). Aunque el bajo rendimiento se asocia con la dificultades económicas a que se ven expuestos los hogares dirigidos 
por mujeres solas, la ayuda pública que puedan recibir no soluciona el problema del bajo rendimiento, dada la baja autoestima de los niños y las bajas perspectivas de los profesores (Dolto, como se cita en Luisi \& Santelices, 2000).

Numerosos estudios muestran que el desarrollo de problemas emocionales y conductuales aumenta en progresión geométrica cuando los niños están expuestos a dos o más factores de riesgo (Doll \& Lyon, 1998; Kolvin et al., 1988).

Si un estudiante pertenece a una familia uniparental y es de nivel socioeconómico y cultural bajo, está en alto riesgo de presentar tanto problemas de rendimiento en la escuela como en sus vivencias personales y sociales, ya que en su medio familiar, escolar y social existen características que lo predisponen a presentar dificultades académicas y personales, dadas las experiencias negativas a las cuales está expuesto.

Sin embargo, los estudiantes también tienen recursos internos que los ayudan o los coartan en su rendimiento académico como la autoestima, las propias expectativas y la motivación intrínseca. Aquellos alumnos que tienen un buen autoconcepto, expectativas positivas respecto a su rendimiento y una motivación intrínseca para aprender consistentemente obtienen más logros en la escuela que aquellos que muestran una autoestima pobre, bajas expectativas y una motivación de logros dominada por los refuerzos extrínsecos (Arancibia, 1996). Actualmente se piensa que el bienestar psicológico está más relacionado con la subjetividad de las personas que con referentes externos (Casullo \& Solano, 2000). 


\section{Cómo disminuir las repercusiones de las transformaciones familiares en los niños}

La tendencia al aumento de familias uniparentales, tanto en Chile como en otros países, hace imperiosa la necesidad de implementar acciones destinadas fundamentalmente a crear redes de apoyo a las familias disfuncionales desde la escuela. Cada vez es más importante el rol de la escuela en el desarrollo personal y de valores de los niños.

Si bien no es posible reemplazar las funciones que tradicionalmente la familia cumplía, al menos es factible, trabajando con los recursos personales internos de los alumnos, disminuir el riesgo de dificultades de rendimiento, de conducta, de problemas emocionales y de deserción, enseñando a los niños a modificar sus sentimientos y así sobrellevar las vicisitudes de la vida.

Existen escenarios sociales donde se aprenden modelos y antimodelos como ocurre a través de las familias funcionales y disfuncionales respectivamente. De la misma forma en que se transmiten, de igual forma se pueden modificar.

En programas de prevención de problemas emocionales, conductuales y de rendimiento asociados a transformaciones familiares es necesario desarrollar en los niños el autoconocimiento y las habilidades emocionales tales como la expresión, el manejo y la evaluación de la intensidad de los sentimientos y una actitud positiva frente a la vida.

Asimismo, los niños necesitan saber que ellos no han sido la causa de la separación y/o de los conflictos entre sus padres. Se les debe asegurar que ambos padres siguen amándolos, se les debe estimular a que expresen sus emociones y sentimientos de temor, tristeza o rabia. Deben aprender también a aceptarse a sí mismos, a verse a sí mismos bajo un prisma positivo, a reconocer sus puntos débiles y sus fortalezas, y a disminuir la depresión, la tristeza y el aislamiento. 
La escuela puede ayudar a que se desarrolle en los niños la resiliencia, esa capacidad de los seres humanos de sobreponerse a los trastornos psicológicos y a las heridas emocionales más graves. Los niños pueden tener un desarrollo psicológico normal a pesar de los factores de riesgo que los rodean.

Es posible implementar talleres de desarrollo personal para los estudiantes cuyas familias sean disfuncionales. El propósito del desarrollo personal es propiciar el autoconocimiento y el de los demás y el fenómeno de interacción humana, lo que conlleva a solucionar ciertas problemáticas del diario vivir. El desarrollo personal, basado en los postulados de la psicología humanista, ha desarrollado su propio enfoque, técnicas y métodos para su orientación y guía, considerada hoy desde una perspectiva científica como una mezcla de terapia y educación del sí mismo con la finalidad de autovalerse y de participar con los demás.

También en estos talleres es necesario afianzar la buena relación que el niño tenga con uno de sus padres o con familiares, quienes ejercen un rol protector ante los riesgos para el desarrollo emocional e intelectual que amenazan a los niños cuyas familias son disfuncionales.

\section{Referencias}

Alvarez, M. (1986). Deprivación y modelos parentales. Santiago: Editorial Universitaria.

Amar, J. \& Abello, R. (1998). El niño y su comprensión del sentido de la realidad. Barranquilla: Uninorte.

Arancibia, V. (1996). Factores que afectan el rendimiento escolar en niños pobres. Santiago: CEPAL.

Arancibia, V., Herrera, P. \& Strasser, K. (1997). Manual de Psicología Educacional. Santiago: Universidad Católica de Chile.

Beech, C. (1985). Learning to read. Nueva York: College Hill Press. 
Broman, S., Bien, E. \& Shaugenessy, P. (1985). Low achieving children. The first seven years. Hillsdale, NJ: Lawrence Erlbaum.

Casullo, M. \& Solano, A. (2000). Evaluación del bienestar psicológico en estudiantes adolescentes argentinos. Revista de Psicología de la Pontificia Universidad Católica del Perú, 18 (1), 35-68.

De Castro, J. (1996). El consorcio familia-escuela católica. Documento presentado en el seminario internacional La Realidad Familiar: Un Desafío Educativo en Latinoamérica. Santiago, Chile.

Doll, B. \& Lyon, M. (1998). Risk and resilience: Implications of the delivery of educational and mental health services in the schools. School Psychology Review, 27 (3), 348-363.

Filp, J. (1995). Éxito y fracaso escolar en los hogares pobres: cómo piensan y actúan las profesoras. En X. Sánchez, F. Fernández \& C. Amtmann (Eds.), Educación y pobreza. Valparaíso: Universidad de Playa Ancha de Ciencias de la Educación.

Flecha, R. \& Tortajada, I. (1999). Retos y salidas educativas en la entrada del siglo XXI. Los retos del futuro inmediato. Barcelona: Biblioteca de Aula.

Florenzano, R. (1998). Familia y salud de los jóvenes. Familia y crisis conyugal. Santiago: Universidad Católica de Chile.

Fuenzalida, A. \& Jiménez, M. (1994). Programa Padres e Hijos. Una experiencia educativa de colaboración entre el jardín infantil y la familia. En B. Icaza \& L. Mayorga (Eds.), Familia, jardín infantil, escuela y aprendizaje. Santiago: CIDE.

Gardner, H. (1995). Inteligencias múltiples. Buenos Aires: Paidós.

Gligo, M. E. (1996). La función educadora de la familia. Revista de Pedagogía, 381, 9-12.

Goleman, D. (1996). La inteligencia emocional. Santiago: Javier Vergara.

Halpern, R. (1986). Effects of early childhood interventions on primary schools progress in Latin America. Comparative Education Review, 30 (2), 193-215.

Hansen, S., Sanders, S., Scott, M. \& Last, C. (1998). Predictors of severity absenteeism in children with anxiety-based school refusal. Journal of Clinical Child Psychology, 27(3), 246-254. 
Hetherington, E. (1980). Children and divorce. En R. Henderson (Ed.), Parent-child interaction: Theory, research and prospect. Nueva York: Academic Press.

Hollsteiner, M. \& Tacon, P. (1983). Urban migrations in developing countries: Consequences on families and their children. En D. Wagner (Ed.), New directions for child development. San Francisco: Jossey Bass.

Iglesias De Ussel, J. (1998). La familia y el cambio político en España. Madrid: Tecnos.

Jackson, Y. \& Frick, P. (1998). Negative life events and adjustments of school-age children: Testing protective models. Journal of Clinical Child Psychology, 27 (4), 370-380.

Jadue, G. (1996a). Características familiares de los hogares pobres que contribuyen al bajo rendimiento o al fracaso escolar de los niños. Revista de Psicología de la Pontificia Universidad Católica del Perú, 14, 35-45.

Jadue, G. (1996b). Efectos de un trabajo con madres de bajo nivel socioeconómico y cultural en el rendimiento escolar de sus hijos. Boletín de Investigación Educacional, 2, 51-63.

Jadue, G. (1999). Hacia una mayor permanencia en el sistema escolar de los niños en riesgo de bajo rendimiento y de deserción. Estudios Pedagógicos, 25, 83-90.

Jadue, G. (2002). Factores psicológicos que predisponen al bajo rendimiento, al fracaso y a la deserción escolar afectan el rendimiento escolar. Estudios Pedagógicos, 28, 193-204.

Johnson, G. M. (1998). Students at risk. School Psychology International, 19, 221-237.

King, N., Mietz, L. \& Ollendick, T. (1995). Psychopathology and cognitions in adolescents experiencing severe test anxiety. Journal of Clinical Child Psychology, 22, 17-27.

Kolvin, J., Miller, F., Fleeting, M. \& Kolvin, P. (1988). Social and parenting factors affecting criminal-offense rates. Findings from the Newcastle Thousand Family Study. British Journal of Psychiatry, 152, 80-90. 
Kotliarenco, M., Cáceres, I. \& Castro, A. (1994). La familia, un puente entre la educación inicial y básica. En B. Icaza \& L. Mayorga (Eds.), Familia, jardín infantil, escuela y aprendizaje (pp. 77-88). Santiago: CIDE.

Kotliarenco, M., Cáceres, I. \& Cortéz, M. (1995). Introducción a las notas sobre la pobreza. CEAMIN. En Aporte de la educación parvularia para la superación de la pobreza. Santiago: JUNJIComisión Nacional de Educación Parvularia.

Lau, S. \& Leung, K. (1992). Relations with parents and school and Chinese self-concept, delinquency and academic performance. British Journal of Educational Psychology, 62, 193-202.

Luisi, V. \& Santelices, L. (2000). Efectos de la separación de los padres en el desempeño escolar de los hijos. Un estudio descriptivo de resultados de investigaciones en las últimas dos décadas. Boletín de Investigación Educacional, 15, 143-151.

Mc Allister, S. (1990). La participación de los padres y su relación con los logros de los niños. Santiago: CIDE.

Mc Lanahan, S. (1985). Family structure and the reproduction of poverty. American Journal of Sociology, 90 (4), 873-901.

Montenegro, H. (1989). Retardo mental sociocultural. Santiago: Universidad Católica de Chile.

Organización de las Naciones Unidas para la Educación, la Ciencia y la Cultura (UNESCO) (1997). Informe de la Comisión Mundial de Cultura y Desarrollo. París, Francia.

Organización de las Naciones Unidas para la Educación, la Ciencia y la Cultura (UNESCO)/Fondo de las Naciones Unidas para la Infancia (UNICEF) (1996). La prioridad es la infancia. Cumplimiento de las metas de la cumbre mundial a favor de la infancia. París, Francia.

Osborn, A. (1990). Resilient children: A longitudinal study of high achieving socially disadvantaged children. Early Child Development and Care, 62, 23-47.

Pitiyanuwat, S. \& Reed, J. (1994). Socioeconomic status as a mayor effect on math achievement, educational aspirations and future job 
expectations in Thailand. International Journal of Educational Research, 21(79), 713-721.

Programa de las Naciones Unidas para el Desarrollo (PNUD). (2000).

Nosotros los chilenos: un desafío cultural. Santiago, Chile.

Ramey, C. \& Campbell, F. (1984). Preventive education from high-risk children: Cognitive consequences of the Carolina Abecedarian Project. American Journal of Mental Deficiency, 88 (5), 515-523.

Rutter, M. (1983). Stress, coping and development in children. Nueva York: Mc Graw Hill.

Salovey, P. \& Sluyter, S. (1997). Emotional development and emotional intelligence. Nueva York: Basic Books.

Santelices, L., Ossandón, L. \& De la Barrera, M. (2000). Diagnóstico preliminar y algunas propuestas para apoyar desde la escuela a los hijos de padres separados. Boletín de Investigación Educacional, $15,152-175$.

Sarquis, C. (1993). Introducción al estudio de la pareja humana. Santiago: Universidad Católica de Chile.

Triandis, H., Bontempo, R., Villareal, M., Asai, M. \& Luca, N. (1988). Individualism and collectivism: Cross-cultural perspectives on selfgroup relationships. Journal of Personality and Social Psychology, 54, 323-338.

Villalón, M., De Castro, J. \& Streeter, B. (1998). El desarrollo de los valores de la verdad y el amor en el ámbito familiar. Boletín de Investigación Educacional, 13, 24-35.

Wang, M. (1995). Atención a la diversidad del alumnado. Madrid: Narcea.

Williams, D. \& Chaukin, N. (1989). Essential elements of strong parent involvement programs. Educational Leader, 47 (2), 1820.

Zimiles, H. \& Lee, V. (1991). Adolescent family structure and educational progress. Developmental Psychology, 27 (2), 314 320. 